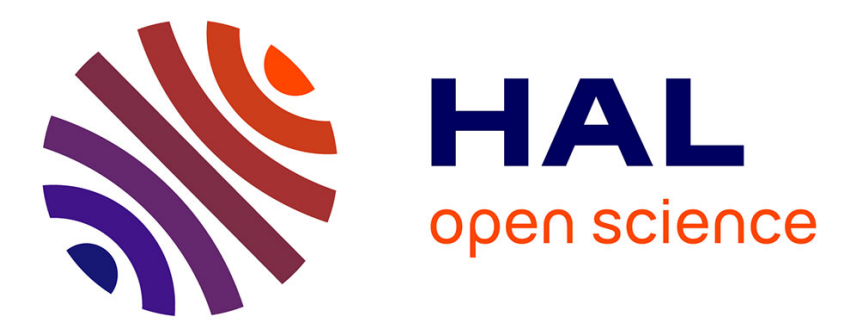

\title{
Synthesis and processing of nanoscaled ceramics by chemical routes
}

H. Schmidt, R. Nass, M. Aslan, K.-P. Schmitt, T. Benthien, S. Albayrak

\section{To cite this version:}

H. Schmidt, R. Nass, M. Aslan, K.-P. Schmitt, T. Benthien, et al.. Synthesis and processing of nanoscaled ceramics by chemical routes. Journal de Physique IV Proceedings, 1993, 03 (C7), pp.C71251-C7-1260. 10.1051/jp4:19937193 . jpa-00251826

\section{HAL Id: jpa-00251826 https://hal.science/jpa-00251826}

Submitted on 1 Jan 1993

HAL is a multi-disciplinary open access archive for the deposit and dissemination of scientific research documents, whether they are published or not. The documents may come from teaching and research institutions in France or abroad, or from public or private research centers.
L'archive ouverte pluridisciplinaire HAL, est destinée au dépôt et à la diffusion de documents scientifiques de niveau recherche, publiés ou non, émanant des établissements d'enseignement et de recherche français ou étrangers, des laboratoires publics ou privés. 


\title{
Synthesis and processing of nanoscaled ceramics by chemical routes
}

\author{
H. SCHMIDT, R. NASS, M. ASLAN, K.-P. SCHMITT, T. BENTHIEN and S. ALBAYRAK \\ Institut für Neue Materialien, Im Stadtwald, Geb. 43, 66123 Saarbrücken, Germany
}

\section{Synthesis and Processing of Nanoscaled Ceramics by Chemical Routes}

\begin{abstract}
Nanocrystalline boehmite and TiN powders have been used as model systems for testing the conception of small-molecule surface modification for the preparation of processable nanoscaled slips and pastes with a high solid content. It was found that after surface modification of boehmite with carboxylic acids like acetic or propionic acid and the addition of 5 to 6 vol.-\% of organic processing additives pastes with a solid content of about 45 vol.- $\%$ can be prepared. The nanocrystalline pastes have been extruded to tubes which after drying exhibit green densities of about 55 to $60 \%$ of the theory. In the case of TiN it was shown that deagglomeration with short-chained organic bases gives low viscous slips with a solid content of 40 .wt. $\%$. Green bodies with a green density of about $50 \%$ of the theory have been prepared by vacuum-assisted pressure filtration. The nanocrystalline material exhibits a very high sintering activity so that a density above $96 \%$ of the theory could be achieved at $1150^{\circ} \mathrm{C}$ leading to a microstructure consisting of an average grain size of about $50 \mathrm{~nm}$. This clearly demonstrates the potential of nanosized starting material for separating the densification regime from the grain growth regime in the sintering step.
\end{abstract}

\section{Introduction}

The question of preparation of ceramic components with a reduced flaw size is still an important feature for structural as well as for functional ceramics. Defects generally result from a variety of sources such as inhomogeneities in the raw materials, coarse particles or hard agglomerates, inhomogeneous pore size distribution or density fluctuations from the green body preparation [1 - 4]. Another type of flaws results from impurities introduced in the ceramic by milling processes or from the atmosphere. For the latter one, clean room technologies or closed system technologies are able to reduce these impurities substantially. Much more serious with respect to defect-avoiding strategies is the "intrinsic" introduction of flaws by the starting material, the powder processing step and the shaping process. For example, density fluctuation in green bodies, as a rule, result in stresses after firing which especially in the case of complex shapes reduce the strength of the ceramic bodies substantially. For these reasons, processing techniques are desirable which, on the one side, may lead to defectfree green bodies and on the other side, can build up defect-healing mechanisms during sintering. 
One basic strategy is to separate the densification regime from the grain growth regime. That means, both processes should take place at different temperatures. One of the potential routes to achieve these goals is the use of nanoscaled raw materials. As shown already with metals [5], particle sizes in the range of several nanometers show a behaviour quite different from larger crystals with respect to diffusion coefficient and densification behaviour. It has also been demonstrated that sol-gel processing of ceramic materials can lead to extremely low densification temperatures especially for thin films or some compact materials [6 - 9]. This can be attributed to the fact that colloidal systems produced by sol-gel processes consist of particles of several to several tens of nanometers in size. Gel pieces consisting of nanosized particles show an excellent sintering behaviour if they possess a homogeneous nanosized pore size distribution. But this does not automatically lead to technically feasible processing routes for compact ceramic materials since it is extremely difficult to produce green bodies with a homogeneous microstructure and a sufficient green density. Large-piece processing is therefore accompanied by the usual difficulties leading to inhomogeneous green bodies by agglomerate formation.

In wet processing, optimized green body compaction requires additives for stabilizing the ceramic slip. These additives additionally act as "lubricants" for reducing particle-particleinteraction and to avoid the formation of agglomerates.

In order to utilize the intrinsic potential of nanoscaled ceramic particles, this conception basically seems to be attractive, but in reality, the transfer of using conventional additives results in difficulties. As demonstrated by Aksay, the use of different types of lubricants is essential for the fabrication of defect-free green bodies with submicron particles [10]. Polymeric materials with different functional groupings (polyphosphates, alcohols, glycols, sulfates) have been used successfully. With particle sizes in the submicrometer range $(100 \leq d<1$ $\mu \mathrm{m})$, this concept is very effective since the additives do not contribute substantially to the green body composition. With reduced particle sizes, the relative amount of additives increases since this amount is proportional to the ceramic particle surface area leading to a permanent decrease of the ceramic content in the green bodies. For this reason, new conceptions have to be developed with the ability to control the particle-particle interaction, to avoid the formation of aggregates and, in addition to this, lead to high green densities. In this paper, a new conception, the so-called small-molecule surface modification, was investigated on two different systems: nanoscaled boehmite and nanoscaled titanium nitride systems.

\section{Experimental}

\section{Aqueous Suspensions of Boehmite}

Suspensions of boehmite (Condea Chemie, FRG) have been prepared by adding $1.5 \mathrm{~kg}$ of the as received powder to $4.350 \mathrm{~kg}$ water and $0.150 \mathrm{~kg}$ acetic and propionic acid, respectively, under stirring. The slurry was refluxed for 20 hours and samples for partricle size measurements have been taken every hour. $1 \mathrm{~g}$ of the suspensions were diluted with 50 $\mathrm{g}$ distilled water and the particle size was determined by photon correlation spectroscopy. For the determination of the viscosity as a function of solid content, water was evaporated step by step in vacuum ( $p \leq 100 \mathrm{mbar}, T=50^{\circ} \mathrm{C}$ ) and viscosity measurements were performed by using a rotary viscosimeter in the shear rate range from $10-1000 \mathrm{~s}^{-1}$.

\section{Preparation of Extrusions Pastes}

For the preparation of extrusion pastes, dry and with propionic acid modified boehmite powder ( 5 wt.-\% acid) was used prepared by the above described procedure. Dry powders 
were obtained by freeze drying after a refluxing time of 15 hours. A kneader was fed with $250 \mathrm{~g}$ modified boehmite powder and $300 \mathrm{~g}$ of distilled water, and this mixture was kneaded at $50^{\circ} \mathrm{C}$ for 30 minutes. 6 to $8 \mathrm{wt} . \%$ of a mixture of a cellulose binder and a polyethylenoxide plasticizer was added and the whole mixture was homogenized by kneading for an additional 0.5 hour. Then water was evaporated in vacuum ( $p \leq 100 \mathrm{mbar}$ ) and the change in viscosity of the pastes was monitored by following the electric current take-up of the kneader. Pastes prepared in this way were used for extrusion experiments by using a piston extruder. As a model geometry a tube with an outer diameter of $2 \mathrm{~cm}$ and a wall thickness of $3 \mathrm{~mm}$ was chosen and tubes $2 \mathrm{~m}$ in length have been extruded. Drying of the tubes was performed in a controlled atmosphere.

\section{Preparation of TiN Slips}

$45 \mathrm{~g} \mathrm{TiN}(\mathrm{H}$. C. Starck, FRG) were dispersed in $55 \mathrm{~g}$ of a water/ethanol mixture under stirring. To this suspension 0.1 to $5 \mathrm{wt} . \%$ of ammonia, tetramethyl ammoniumhydroxid, guanidine chloride and guanidine carbonate with regard to the solid content were added and refluxed for 4 hours. After cooling to room temperature the slips were ultrasonically agitated, sieved and used for further experiments. The particle size of the dispersed TiN has been measured by photon correlation spectroscopy and TEM.

\section{Preparation of TiN Specimens}

Following the above described procedure an ethanolic slip with a solid content of $40 \mathrm{wt.} . \%$ TiN powder was prepared by using $2 \mathrm{wt.} \%$ of guanidine carbonate with respect to the solid content. From this slip green disks $(5 \mathrm{~cm}$ in diameter, $3 \mathrm{~mm}$ in thickness) have been produced by vacuum-assisted pressure filtration. The obtained green bodies were dried first for 24 hours in a saturated ethanolic atmosphere using a desiccator. The drying procedure was completed by drying the green specimen in an oven at $70^{\circ} \mathrm{C}$. Sintering was performed at temperatures between $1000^{\circ} \mathrm{C}$ and $1300^{\circ} \mathrm{C}$ for 30 minutes unter flowing argon.

\section{Results and Discussions}

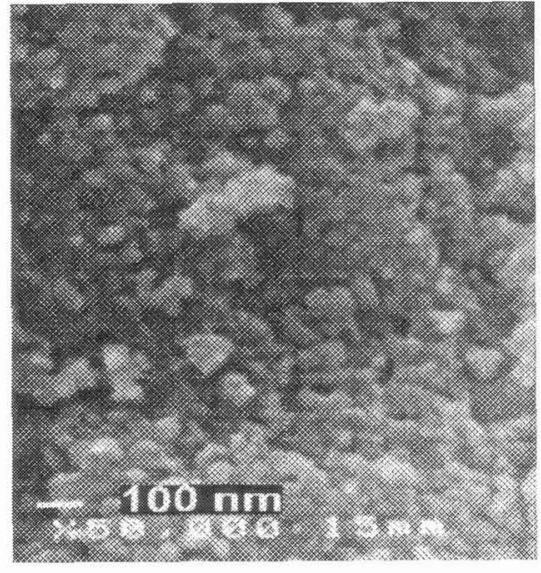

Fig. 1: SEM picture of the boehmite powder as received.
Bohemite powders are commercially available from different companies. In general, these powders consist of strongly agglomerated nanocrystalline particles ranging in size from 5 to 20 nanometers. This results from the precipitaiton process during which the nanoscaled particles are growing together by chemical bonds, forming strong agglomerates. In figure 1 a SEM picture of the as received powder is shown.

It is quite obvious that the agglomerates exhibit a globular microstructure consisting of nanosized primary particles less than $20 \mathrm{~nm}$ in diameter. However, this boehmite can not be deagglomerated to its primary particle size by conventional surface active compounds because they are not able to break the interparticle necks. In order to break the necks, boehmite powder has been treated with 
different organic acids such as acidic and propionic acid in aqueous solution under reflux conditions. The particle size of the suspension has been followed by photon correlation spectroscopy. In figure 2 the particle size reduction is shown as a function of refluxing time for the boehmite/ propionic acid system.

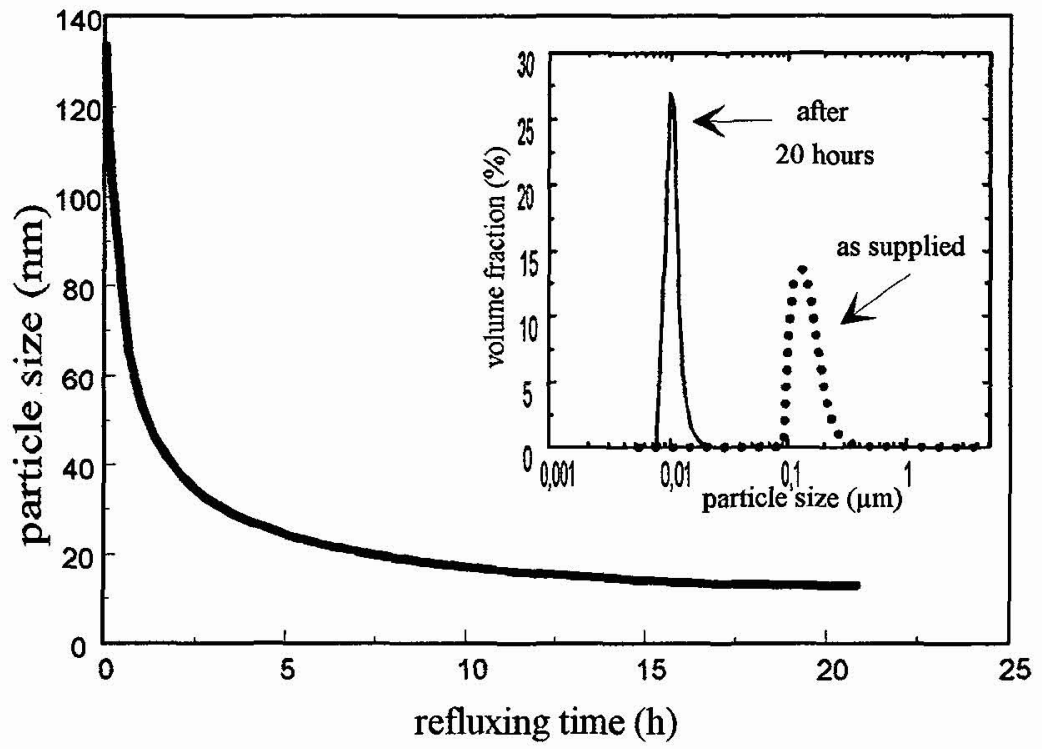

Fig. 2: Reduction of particle size of the as received boehmite powder as a function of refluxing time treated with $5 \mathrm{wt.}$.\% propionic acid. For comparison, the particle size distribution at the beginning and the end of the refluxing procedure is shown, too.

Figure 2 shows that the acid treatment results in a continuous decrease of the size of the suspended particles with increasing refluxing time and that after 15 hours the primary particle size of approximately $15 \mathrm{~nm}$ is achieved as it could be expected from the microstructure shown in fig. 1. In fig. 3 a TEM micrograph of the fully dispersed boehmite powder is shown.

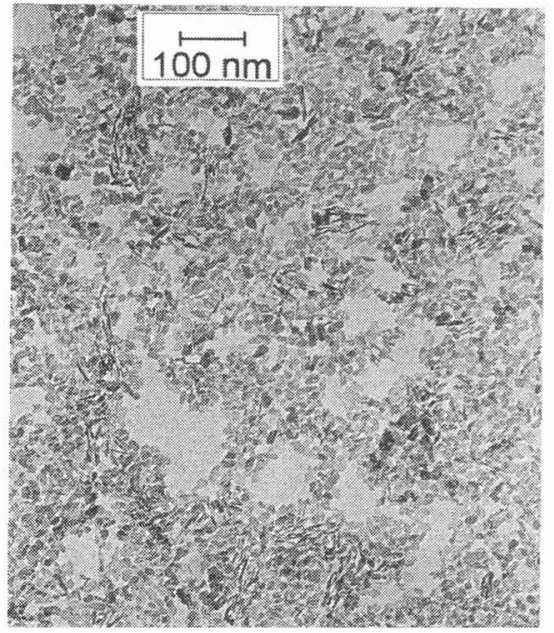

The powders were separated from the acid by centrifugation, subsequently washed with ethanol and dried in a desiccator. The question arises whether these powders still contain acidic or propionic acid or not. For this reason, DRIFT (Diffuse Reflection Infrared Fourier Transform) spectroscopy measurements have been carried out. In fig. 4, the infrared spectrum of the powder modified with 5 wt.-\% of propionic is shown and compared with the spectrum of the free acids.

Fig. 3: TEM picture of a boehmite powder dispersed with propionic acid in water after a refluxing time of 16 hours. 


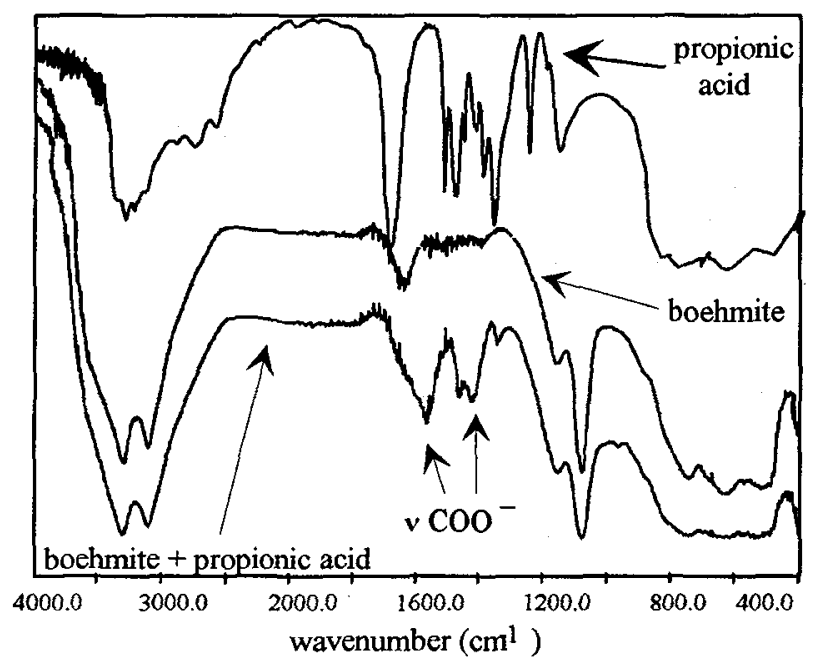

Fig. 4: Comparison of free carboxylic acid, as received boehmite powder and surface modified boehmite by IR spectroscopy.

As one can see clearly, the dried powders still contain the acid groupings but the shift of the $\mathrm{C}=\mathrm{O}$ frequency to the salt form indicate that a surface compound has been formed whose structure is very similar to the alumina salts of the used acids.

This is a very interesting effect since it indicates that a very strong interaction between the acids and the boehmite surface takes place which "survives" the removal of the aqueous phase with the dissolved acid. Since the space required by the acetate or propionate groups is in the range of a few $\mathrm{A}^{2}$, the model which can be built up resembles the conventional ceramic powder/additive model but now projected from the micrometer range into the nanometer range.

In order to get data about the amount of acid adsorbed at the powder surface, the adsorption behaviour was investigated. The results are shown in fig. 5 .

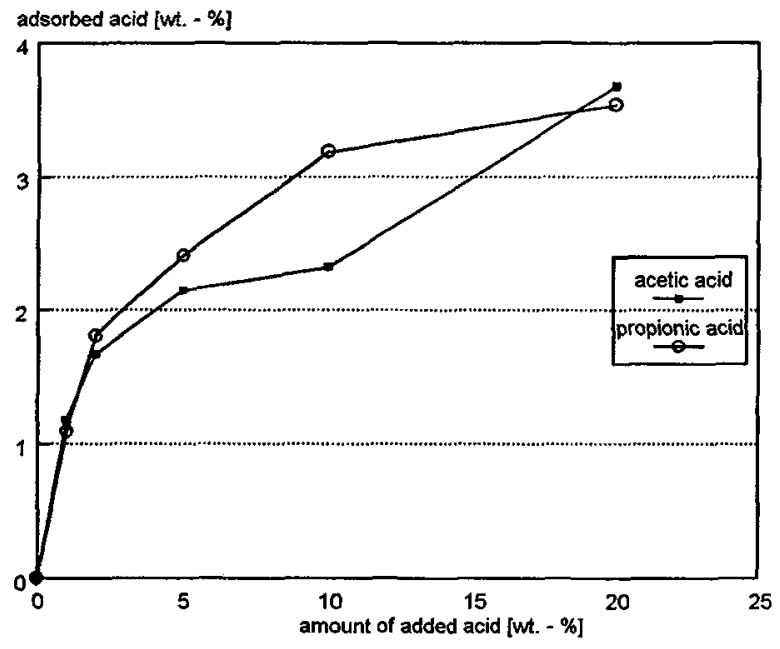

Fig. 5: Adsorption behaviour of acetic and propionic acid on nanoscaled boehmite powder. 
As one can conclude from the adsorption, there is a saturation of the surface after the absorption of 2.5 to $3.0 \mathrm{wt} . \mathrm{-} \%$ of propionic or acetic acid. Assuming that propionic acid molecule requires a space of about $0.35 \mathrm{~nm}^{2}$ (calculated from incremented data of bond lengths and angles) and the boehmite consists of particles with an average diameter of about $15 \mathrm{~nm}$, the amount of propionic acid adsorbed in a monolayer can roughly be calculated. From these data a theoretical value of about $6 \mathrm{wt} . \%$ is obtained. Comparing the theoretical coverage with the experimental value of 3 to $3.5 \mathrm{wt} . \%$, one can conclude that the surface probably contains still a reasonable amount of $\mathrm{OH}$ groups, responsible for the good dispersibility of the system in aqueous phases. A complete coverage of the surface with propionic or acetic acid groupings should lead to a change of polarity and result in hydrophobic particles. The results lead to a model shown in fig. 6 for the surface modification of nanocrystalline boehmite to particles.
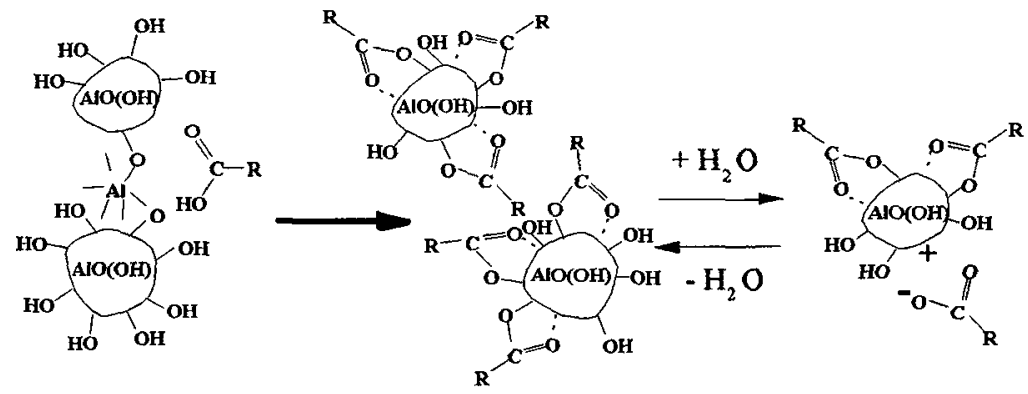

$$
\mathrm{R}:-\mathrm{CH}_{3}, \mathrm{CH}_{2} \mathrm{CH}_{3}, \ldots
$$

Fig. 6: Model of the surface modification of nanosized boehmite particles by carboxylic acids.

It was of further interest how far this surface modification actually can reduce the particleparticle interaction and how far this can be used to increase the solid content of dispersed systems. As shown above, the particle size is $15 \mathrm{~nm}$ in average, which is typical for alumina sols electrostatically stabilized with inorganic acids like nitric or hydrochloric acid. Using such sols, solid contents of about 10 to a maximum of $20 \mathrm{wt} . \%$ can be obtained. Increasing the solid content leads to rigid gels. In the following the viscosity of the dispersions was investigated as a function of solid content for boehmite sols treated with $5 \mathrm{wt} . \%$ acetic and propionic acid, respectively. The results are shown in fig. 7 .

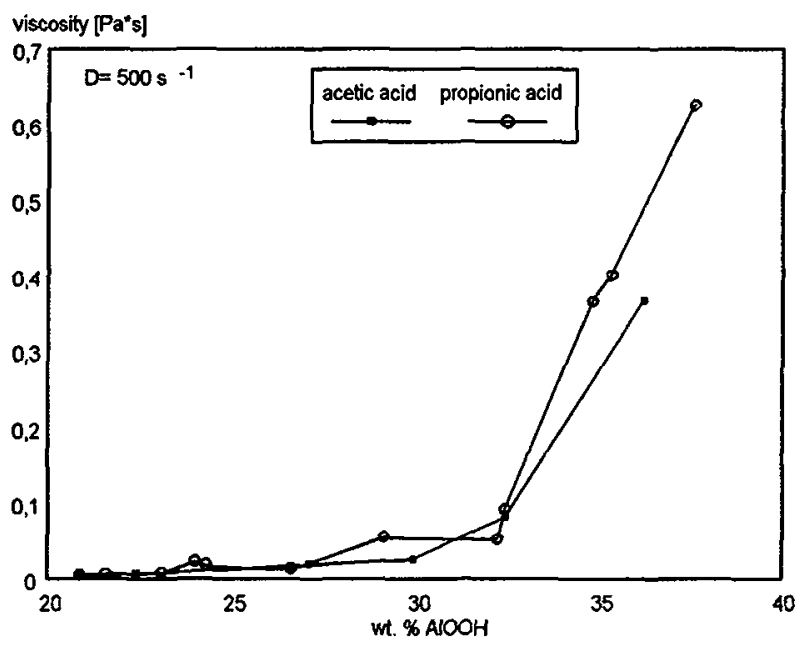

Fig. 7: Viscosity of boehmite sols treated with 5 wt. $-\%$ acetic and propionic acid, respectively, at $100^{\circ} \mathrm{C}$ for 15 hours. 
It can be seen that solid contents between 35 and 38 wt.-\% can be obtained and that the viscosity of these sols at a shear rate of $500 \mathrm{~s}^{-1}$ in the range of 300 to $600 \mathrm{mPa} \cdot \mathrm{s}$. Shear stress/shear rate measurements further show an newtonian flow behaviour which indicates a good state of dispersion free of particle-particle interaction. The solid content of these sols was further increased by evaporating water in a vacuum kneader. In the case of the propionic acid stabilized powder a paste with a solid content of about 32 vol.-\% could be fabricated. The paste is highly transparent, and due to its plastic flow behaviour, it can be used in extrusion processes. In order to obtain sufficient green strength and to improve the extrusion behavior, organic polymers were added in overall concentrations of less than 8 wt.$\%$. In fig. 8 the torque of the pastes as a function of the solid content for two different concentrations of organic additives is given.

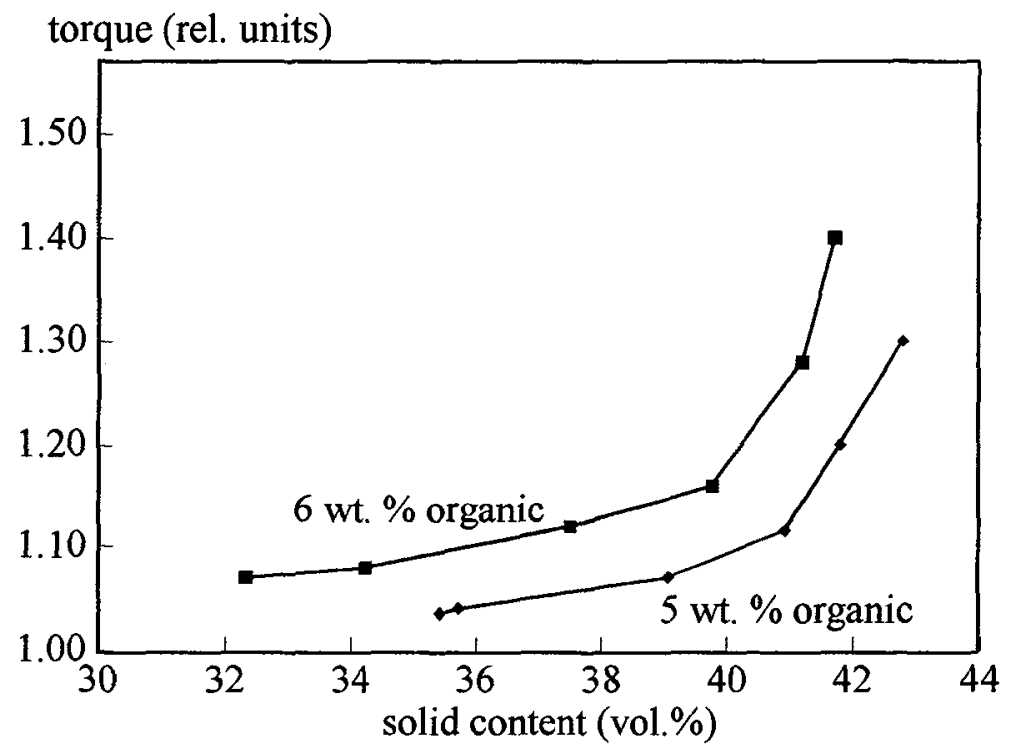

Fig. 8: Torque of the propionic acid modified pastes as a function of the solid content and 5 and $6 \mathrm{wt}$. $\%$ organic additives, respectively.

By this means, the solid content of the sols could be increased up to values between 40 and 42 vol.-\% depending on the amount and composition of the additional organic processing additives. It is reasonable that the viscosity with $5 \%$ organic is lower than that with $6 \%$ organic. This phenomenon seems to indicate an optimum organic concentration, but has to be investigated in more detail.

It is notable that the relatively low viscosity of the system is not based on the rheological properties of polymers as it is the case of ceramic extrusion processes not based on clay minerals, and furthermore, it is notable that with an average particle size of $15 \mathrm{~nm}$, the interaction is comparingly low. As shown in a variety of literature [11 - 17] systems with solid contents in this range are not obtainable in solid bulk components. From these pastes tubes can be extruded (fig. 9) which are flexible after extrusion and can be fired to porous alumina tubes between 1100 and $1300^{\circ} \mathrm{C}$. 


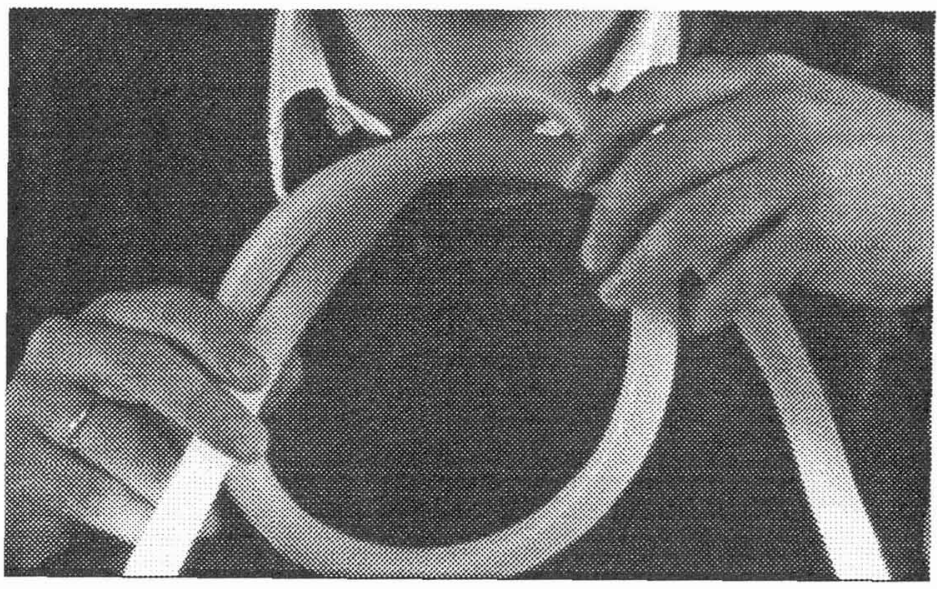

Fig. 9: Extruded boehmite gel tube.

The results show that the conception of small molecule surface modification can be used for the nanoprocessing of boehmite. This system has been used as a model system since boehmite is commercially available, and, after solving the problem of hard agglomerates, can be used as a cheap source for $5-20 \mathrm{~nm}$ boehmite particles.

Subsequently it was tried to use this conception for nonoxide ceramics, too. For this purpose, titaniumnitride powder produced by a chemical vapour reaction process (CVR, $H$. C. Starck company) was investigated. Again surface modification has been employed to get a high degree of dispersion, and in fig. 10 the effect of different dispersing agents on the deagglomeration of TiN is shown.

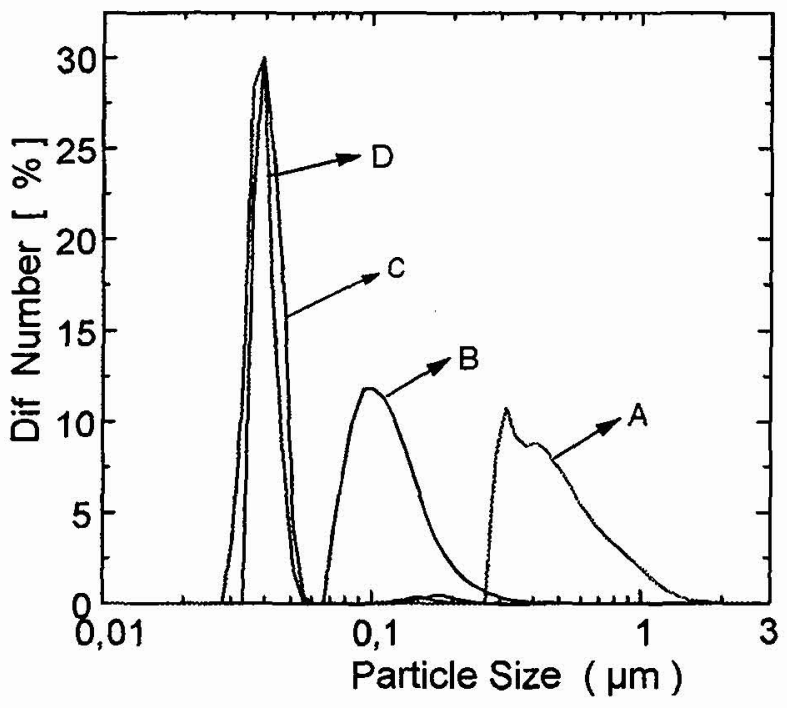

Fig. 10: Particle size distribution of nanoscaled TiN slips stabilized with different dispersion agents. A: ammonia, B: amino propionic acid, C: guanidine propionide acid, D: guanidine carbonate. 
As one can see, the use of guanidino compounds, especially guanidine carbonate, leads to the deagglomeration into the primary particles 20 to $50 \mathrm{~nm}$ in diameter as shown in fig. 11 .

The nanodispersed slip then was shaped by a pressure filtration process into disks of about $5 \mathrm{~cm}$ in diameter and $3 \mathrm{~mm}$ thickness which have been sintered at $1150^{\circ} \mathrm{C}$ to densities exceeding $96 \%$ of theoretical value. In fig. 12 , the microstructure of a fracture surface of the sintered material without machining is shown.

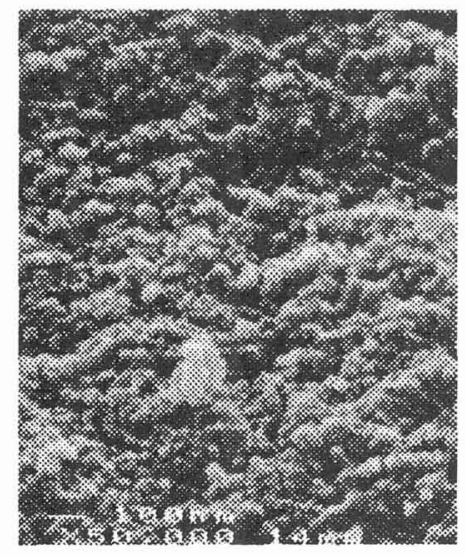

Fig. 11: Microstructure of a TiN-specimen sintered at $1150^{\circ} \mathrm{C}$

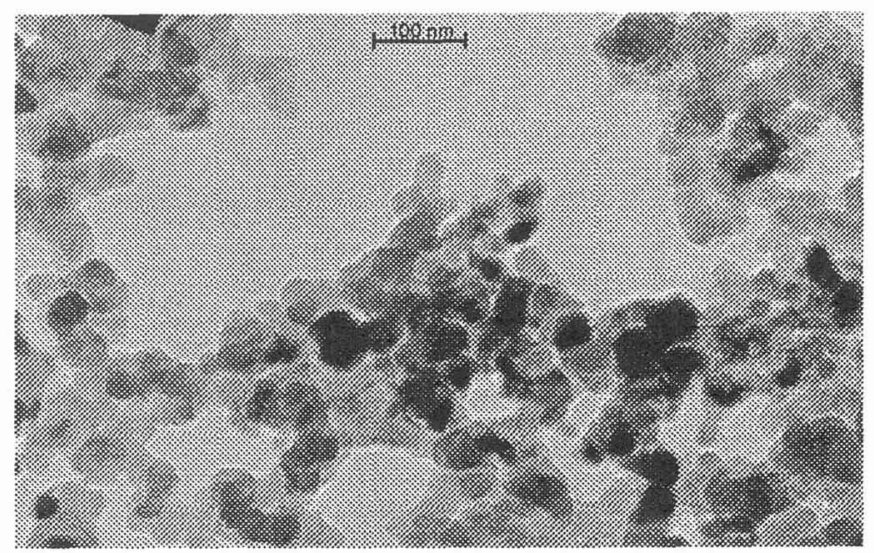

Fig. 12: TEM picture of the TiN powder deagglomerated with guanidine carbonate.

It was found that the sintering process takes place without substantial grain growth, and a nanoscaled microstructure can be obtained. This clearly demonstrates that densification and grain growth can be separated by using nanoscaled ceramic powders.

Determination of the hardness of sintered specimens with a relative density of $96 \%$ gave a $\mathrm{HV}_{0.1}$ value of $21.5 \mathrm{GPa}$. Taking into account that conventional TiN exhibits a hardness of $H V_{0.1}=20.6 \mathrm{GPa}$ if the density exceed $99 \%$ of the theory, the hardness of the less dense nanostructured ceramic is quite high.

\section{Conclusion}

It can be concluded that nanoscaled ceramic particles can be processed to high solid content green bodies, if the surface interaction of the small particles can be controlled. Further results have shown that the discussed potential of low sintering temperatures actually can be realized if low-defect green structures can be obtained. This opens routes to nanoprocessed ceramics, which then have to be tested for their structural and functional properties.

\section{Acknowledgement}

The authors want to thank $\mathrm{H}$. C. Starck for their supply with TiN powders as well as the Minister for research and culture of Saarland for the financial support. 


\section{References}

[1] Rice, R. W., in "Processing of Crystalline Ceramics, H. Polmour III, R. F. Davis and F. M. Hase (eds.) Plenum, New York 1978, p. 303 - 322.

[2] Lange, F. F., J. Am. Ceram. Soc. 66, (1983) 396 - 398.

[3] Lange, F. F., J. Am. Ceram. Soc. 66, (1983) $398-406$.

[4] Lange, F. F., Davis, B. I., and Aksay, I. A., J. Am. Ceram. Soc. 66 (1983) $407-408$.

[5] Gleiter, H., Progress in Materials Science 33 (1989) $223-315$.

[6] Messing, G. L., Shelleman, R. A., Kumagai, M., J. Non-Cryst. Solids $\underline{82}$ (1986) $277-285$.

[7] Tomandl, G., Stiegelschnitt, A., Boehner, R., Sci. Ceram. 14 (1988) 305.

[ 8] Chen, C. J., Xu, Y., Xu, R., Mackenzie, J. D., paper presented at 1 st Internat. Cer. Sci. and Tech. Cong., Anaheim, 1989

[ 9] Hirano, S., Kato, K., J. Non-Cryst. Solids 100 (1988) 538.

[10] Aksai, I. A., Stangle, G. C., Sarikaya, M., in: Ceramic Powder Processing Science. Eds.: Hausner, H., Messing, G. L., Hirano, S.; Deutsche Keramische Gesellschaft 1989, 463.

[11] Brinker, C. J., Clark, D. E., Ulrich, D. R. (eds.),

Better Ceramics Through Chemistry I, in: Materials Research Society Symposia Proceedings, Vol. 32, North Holland, New York/Amsterdam/Oxford, 1984.

[12] Brinker, C. J., Clark, D. E., Ulrich, D. R. (eds.),

Better Ceramics Through Chemistry II, in: Materials Research Society Symposia Proceedings, Vol. 73, Materials Research Society, Pittsburgh/PA, 1986.

[13] Brinker, C. J., Clark, D. E., Ulrich, D. R. (eds.),

Better Ceramics Through Chemistry IIII, in: Materials Research Society Symposia Proceedings, Vol. 121, Materials Research Society, Pittsburgh/PA, 1988.

[14] Zelinski, B. J. J., Brinker, C. J., Clark, D. E., Ulrich, D. R. (eds.), Better Ceramics Through Chemistry IV, in: Materials Research Society Symposia Proceedings, Vol. 180, Materials Research Society, Pittsburgh/PA, 1990.

[15] Vilminot, S., Naß, R., Schmidt, H. (eds.), Eurogel ' 91 - Progress in Research and Development of Processes and Products from Sols and Gels, in: European Materials Research Society Monographs, Vol. $\underline{5}$, North Holland/Amsterdam/Londonl/New York/Tokyo, 1992.

[16] Aegerter, M. A. (ed.), Glasses and Ceramics from Gels - Proceedings of the Fifth Int. Workshop on Glasses and Ceramics from Gels. North Holland, Amsterdam, 1990.

[17] Esquivias, L. (ed.), Advanced Materials from Gels - Proceedings of the Sixth Int. Workshop on Glasses and Ceramics from Gels. North Holland, Amsterdam, 1992. 